\title{
Tests for cointegration with infinite variance errors
}

\author{
Mehmet Caner* \\ Department of Economics, Koc University, Cayir Cad No. 5, Istinye 80860, Istanbul, Turkey
}

Received 1 December 1995; received in revised form 1 September 1997

\begin{abstract}
This paper develops the asymptotic theory for residual-based tests and quasi-likelihood ratio tests for cointegration under the assumption of infinite variance errors. This article extends the results of Phillips and Ouliaris (1990) and Johansen $(1988,1991)$ which are derived under the assumption of square-integrable errors. Here the limit laws are expressed in terms of functionals of symmetric stable laws rather than Brownian motion. Critical values of the residual-based tests of Phillips and Ouliaris (1990) and likelihoodratio-based tests of Johansen (1991) are calculated and tabulated.

We also investigate whether these tests are robust to infinite variance errors. We found that regardless of the index of stability $\alpha$, the residual-based tests are more robust to infinite variance errors than the likelihood-ratio-based tests. (C) 1998 Elsevier Science S.A. All rights reserved.
\end{abstract}

JEL classification: $\mathrm{C} 32$

Keywords: Symmetric stable process; Size distortion; Quadratic variation

\section{Introduction}

A considerable literature has developed in the recent years for the analysis of cointegration, see e.g. Engle and Granger (1987), Phillips and Ouliaris (1990), Phillips and Hansen (1990), Johansen (1988, 1991), Johansen and Juselius (1990), Hansen (1992). The commonly used tests for cointegration are the residualbased $\hat{Z}_{\rho}$ and $\hat{Z}_{t}$ tests of Phillips and Ouliaris (1990) and the likelihood-based trace and maximum eigenvalue statistics of Johansen $(1988,1991)$. The distribution theory used to evaluate these test statistics is based on the assumption of square integrable disturbances.

* Now with Bilkent University, Department of Economics, Ankara, Turkey. 
In this paper we investigate whether these tests are robust to infinite variance errors. It may not be immediately obvious that in economics we can find variables with infinite variance. Mandelbrot $(1963,1967)$ shows that certain commodity prices have large variances so that it may be assumed infinite. Fama (1965) notes that the empirical distributions of stock price changes are leptokurtic, and they are governed by stable laws. Recently, McCulloch (1996a,b) argued that financial asset returns are the cumulative outcome of the economic agents decision over time and finds that the returns of the stock prices can be governed by stable laws.

There also have been two recent studies using developing country data sets. Koedijk and Kool (1992) investigate the empirical distribution of black-market exchange rate returns for seven East European currencies focusing on the tails of the distribution. Their results support the existence of finite second moments in exchange rates only for 4 of the seven countries. Another study that examines the black-market exchange rates for 12 Latin American countries is by Akgiray et al. (1988). Strong evidence is found to support the infinite variance hypothesis. This may be the case because of adverse balance of payments, exchange rate and capital controls, large budget deficits. They then argue that constructing a foreign exchange rate position for a country given the nonexistence of a variance might not be easy. These results show us that allowing for infinite variance processes in economic analysis may be prudent.

The aim of our paper is to develop the asymptotic distribution of the residualbased and quasi-likelihood-based tests for cointegration under the assumption of weakly dependent errors with infinite variance. We find that the limit law for the $\hat{Z}_{\rho}$ and $\hat{Z}_{t}$ tests of Phillips and Ouliaris (1990) and the trace and maximum eigenvalue test statistics of Johansen $(1988,1991)$ under infinite variance errors not only consist of functionals of symmetric stable laws but, in the case of Johansen (1991), also involves the quadratic variation of a symmetric stable process. The limit laws depend on the index of stability $\alpha$ and the number of variables in the system. This work extends the results in Phillips and Ouliaris (1990) and Johansen $(1988,1991)$, which are derived under the assumption of finite variance errors, to the case of infinite variance errors.

Since the tests for cointegration depend on the assumption of square-integrable disturbances, it is unknown whether these tests are robust to infinite variance errors or not. We answer this question by calculating the size distortion induced by mistakenly using the conventional critical values of Phillips and Ouliaris (1990) and Johansen $(1988,1991)$. We find that the Phillips and Ouliaris (1990) $\hat{Z}_{\rho}$ and $\hat{Z}_{t}$ tests for cointegration are more robust to infinite variance errors than the Johansen tests. The size distortion that is found biases the tests towards the alternative hypotheses of cointegration. These findings can be important in the empirical research. For example, if we would like to test whether the purchasing power parity holds between two Latin American countries and if we conduct cointegration tests the results may be different depending on the variance of the errors. 
It can be argued that cointegration tests-based on $\mathrm{M}$ - estimators may be more efficient than OLS-based tests, but the scope of this paper is different. We analyze the robustness of the existing cointegration tests to the assumption of infinite variance errors.

In Section 2 some definitions, assumptions and limit theorems that will be useful in the other sections are presented. Section 3 develops the limit theory for the residual-based tests for cointegration and calculates the size distortions when the errors have infinite second moments. In Section 4, the asymptotic distribution theory for quasi-likelihood-based tests for cointegration are derived when the errors have infinite variance. Also, the size distortions which occur by mistakenly using the critical values of the tables in Johansen and Juselius (1990) are calculated. Section 5 extends Section 4's results to the limit law for the trace and maximum eigenvalue statistics with linear trend. Section 6 concludes.

The following notation is used throughout the paper. The symbol ' $\equiv$ ' denotes equality in distribution. $\|A\|=\left[\operatorname{tr}\left(A^{\prime} A\right)\right]^{1 / 2}$ denotes the Euclidean norm of a matrix $A$. ' $\Rightarrow$ ' denotes the weak convergence with respect to the Skorohod metric. $\int A$ is integral with respect to the Lebesque measure. The proofs of the theorems can be obtained from the author on demand.

\section{Preliminaries}

Let $Y_{t}$ be a $p$-vector integrated process generated by the following:

$$
Y_{t}=Y_{t-1}+\xi_{t}, \quad t=1,2, \ldots, n,
$$

where $Y_{0}$ is any random $p$-vector. $\xi_{t}$ is the following linear process:

$$
\xi_{t}=C(L) \varepsilon_{t}
$$

where $C(L)=\sum_{j=0}^{\infty} C_{j} L^{j}$, the $C_{j}$ are $p \times p, C_{0}=I, C(1) \neq 0$ and $C(1)$ is full rank. Also, define

$$
\tilde{C}_{j}=\sum_{v=j+1}^{\infty} C_{v}
$$

Assumption 1. $\varepsilon_{t}$ is iid, symmetrically distributed and in the normal domain of attraction of a $p$-variate symmetric stable law $\mathrm{F}$ with the same index $\alpha$, for all components of the vector $0<\alpha<2 . \varepsilon_{t}$ has independent components. The coefficient matrices $C_{j}$ satisfy the following:

$$
\sum_{j=0}^{\infty} j^{2}\left\|C_{j}\right\|^{\theta}<\infty \quad \text { with } 0<\theta<\alpha \wedge 1 .
$$


$\varepsilon_{t}$ is in the normal domain of attraction of a stable law and we denote that as $\varepsilon_{t} \in \mathscr{N} \mathscr{D}(\alpha)$. This shows that $\alpha$ th moment of the errors is infinite for $\alpha<2$.

Assumption 1 implies the following limit theorems that will be used throughout the paper.

$$
\begin{aligned}
& a_{n}^{-1} \sum_{i=1}^{[n t]} \varepsilon_{i} \Rightarrow Y_{\alpha}, \\
& a_{n}^{-2} \sum_{i=1}^{[n t]} P_{i-1} \varepsilon_{i}^{\prime} \Rightarrow \int_{0}^{t} Y_{\alpha}^{-} \mathrm{d} Y_{\alpha}^{\prime}, \\
& n^{-1} a_{n}^{-2} \sum_{i=1}^{[n t]} P_{i-1} P_{i-1}^{\prime} \Rightarrow \int_{0}^{t} Y_{\alpha}^{-} Y_{\alpha}^{-\prime}, \\
& a_{n}^{-2} \sum_{i=1}^{[n t]} \varepsilon_{i} \varepsilon_{i}^{\prime} \Rightarrow Y_{\alpha}(t) Y_{\alpha}(t)^{\prime}-\int_{0}^{t} Y_{\alpha}^{-} \mathrm{d} Y_{\alpha}^{\prime}-\int_{0}^{t} \mathrm{~d} Y_{\alpha} Y_{\alpha}^{-\prime} \equiv\left[Y_{\alpha}, Y_{\alpha}\right]_{t}, \\
& a_{n}^{-2} \sum_{t=1}^{n} Y_{t-1} \xi_{t}^{\prime} \Rightarrow C(1) \int_{0}^{1} Y_{\alpha}^{-} \mathrm{d} Y_{\alpha}^{\prime} C(1)^{\prime}+\sum_{j=0}^{\infty} C_{j}\left[Y_{\alpha}, Y_{\alpha}\right]_{1} \widetilde{C}_{j}^{\prime}, \\
& n^{-1} a_{n}^{-2} \sum_{t=1 n} Y_{t-1} Y_{t-1}^{\prime} \Rightarrow C(1) \int_{0}^{1} Y_{\alpha}^{-} Y_{\alpha}^{-C^{\prime}} C(1)^{\prime},
\end{aligned}
$$

where $P_{t-1}=\sum_{i=1}^{t-1} \varepsilon_{i}$ and $Y_{\alpha}^{-}$is a symmetrically stable random process with independent components and $\alpha$ is the index of stability. Furthermore, the superscript on $Y$ shows the left limit of the process. $Y_{\alpha}^{-}$belongs to the space of cadlag functions. From here on all the $Y_{\alpha}^{-}$'s on the limits are represented as $Y$ for notational convenience. Moreover, all the limits are on the space of cadlag functions. $\left[Y_{\alpha}, Y_{\alpha}\right]_{t}$ represents the quadratic variation of $Y_{\alpha}(t)$. These results are proved in Sections 3 and 4 of Caner (1997).

We can assume

$$
a_{n i}=a n^{1 / \alpha} \quad \forall i=1,2, \ldots, k \text { for some } \mathrm{a} \neq 0 .
$$

This assumption greatly simplifies the asymptotic theory even though it is restrictive (see Phillips (1995), Section 6). This does not change any of the results obtained, but in order to understand the connections between the limit laws when the errors have finite and infinite variance better, we use Assumption 1. We define the stable process in the following way.

Definition 1. Stable processes are stochastic processes whose sample paths are in $D[0,1]$ with stationary independent increments. These increments have stable distribution. 
For more information on stable processes see chapters 1 and 2 of Samorodnitsky and Taqqu (1994) and Resnick (1986).

\section{Residual-based tests}

First partition $Y_{t}=\left(Y_{1 t}, Y_{2 t}^{\prime}\right)^{\prime}$ into the scalar $Y_{1 t}$ and $m$ vector $Y_{2 t}(m=p-1)$. Then consider the system of cointegrating OLS regressions

$$
\begin{aligned}
& Y_{1 t}=\hat{\beta}^{\prime} Y_{2 t}+\hat{u}_{t}, \\
& \hat{u}_{t}=\hat{\rho} \hat{u}_{t-1}+\hat{e}_{t},
\end{aligned}
$$

where $\hat{\beta}$ and $\hat{\rho}$ are the least-squares estimates and $\hat{u}_{t}$ and $\hat{e}_{t}$ are the regression residuals from Eqs. (11) and (12), respectively.

In this section we develop the limit theory of residual-based tests for cointegration when the error terms have infinite variance. In these tests the null hypothesis is: the residuals $\hat{u}_{t}$ have a unit root. So the null hypothesis corresponds to the hypothesis of no cointegration. Specifically, we analyze the limit behavior of the $\hat{Z}_{\rho}$ and $\hat{Z}_{t}$ tests proposed by Phillips (1987). In the case of finite variance errors, Phillips and Ouliaris (1990) found that the asymptotic distribution only depends on the dimension $p$ of the vector $\varepsilon_{t}$. Our goal is to find the analog for the stable case with $\alpha<2$. We start with a technical result which is immediate from Eq. (10) and helps to prove the main theorems of this section.

Lemma 1. Let

$$
\begin{aligned}
\mathrm{X}_{\alpha} & =C(1) Y_{\alpha} \\
& =\left[\begin{array}{c}
X_{\alpha 1} \\
X_{\alpha 2}
\end{array}\right] .
\end{aligned}
$$

If $Y_{t}$ is generated by Eq. (1), under Assumption 1

$$
\begin{aligned}
\widehat{\beta} & \Rightarrow\left(\int_{0} X_{\alpha 2} X_{\alpha 2}^{\prime}\right)^{-1}\left(\int_{0}^{1} X_{\alpha 2} X_{\alpha 1}\right) \\
& =A_{22}^{-1} a_{21}
\end{aligned}
$$

where

$$
A=\left[\begin{array}{ll}
a_{11} & a_{21}^{\prime} \\
a_{21} & A_{22}
\end{array}\right]=C(1) \int_{0}^{1} Y_{\alpha} Y_{\alpha}^{\prime} C(1)^{\prime}=\int_{0}^{1} X_{\alpha} X_{\alpha}^{\prime} .
$$


$a_{21}$ is an m-dimensional vector and $A_{22}$ is an $m \times m$ full rank matrix and $X_{\alpha 1}$ is a stable process and $X_{\alpha 2}$ is an m-dimensional $\alpha$-stable vector.

This lemma is a generalization of Theorem 2a of Phillips (1986) to the case of infinite variance errors. By Lemma 1 we have

$$
\hat{b}^{\prime}=\left(1,-\hat{\beta}^{\prime}\right) \Rightarrow \eta^{\prime}=\left(1,-a_{21}^{\prime} A_{22}^{-1}\right),
$$

where $\hat{b}$ and $\eta$ are $p$ vectors. Now, set up

$$
\begin{aligned}
& Y_{\alpha}=\left[\begin{array}{l}
Y_{\alpha 1} \\
Y_{\alpha 2}
\end{array}\right], \\
& F=\left[\begin{array}{ll}
f_{11} & f_{21}^{\prime} \\
f_{21} & F_{22}
\end{array}\right] \equiv \int_{0}^{1} Y_{\alpha} Y_{\alpha}^{\prime}, \\
& \kappa^{\prime}=\left(1,-f_{21} F_{22}^{-1}\right), \\
& Q_{\alpha}(t)=Y_{\alpha 1}(t)-\left(\int_{0}^{1} Y_{\alpha 1}(t) Y_{\alpha 2}(t)^{\prime}\right)\left(\int_{0}^{1} Y_{\alpha 2}(t) Y_{\alpha 2}(t)^{\prime}\right)^{-1} Y_{\alpha 2}(t),
\end{aligned}
$$

where $Y_{\alpha 1}$ is a symmetric stable process and $Y_{\alpha 2}$ is an $m \times 1$ symmetric stable vector process with independent components, $f_{11}$ is a scalar, $f_{21}$ is an $\mathrm{m}$ dimensional vector, $F_{22}$ is an $m$-dimensional square matrix.

The limit theory of the following residual-based tests for cointegration can be derived using Eqs. (11) and (12).

1. Phillips $\hat{Z}_{\rho}$ test:

$$
\hat{Z}_{\rho}=n(\hat{\rho}-1)-(1 / 2)\left(s_{n l}^{2}-s_{n}^{2}\right)\left[n^{-2}\left(\sum \hat{u}_{t-1}^{2}\right)\right]^{-1},
$$

2. Phillips $\hat{Z}_{t}$ test:

$$
\hat{Z}_{t}=\left(\sum \hat{u}_{t-1}^{2}\right)^{1 / 2}(\hat{\rho}-1) / s_{n l}-(1 / 2)\left(s_{n l}^{2}-s_{n}^{2}\right)\left[s_{n l}\left(n^{-2} \sum \hat{u}_{t-1}^{2}\right)^{1 / 2}\right]^{-1},
$$

where

$$
\begin{aligned}
& s_{n}^{2}=n^{-1} \sum \hat{e}_{t}^{2}, \\
& s_{n l}^{2}=n^{-1} \sum \hat{e}_{t}^{2}+2 n^{-1} \sum_{s=1}^{l} w_{s l} \sum_{t=1}^{n-s} \hat{e}_{t} \hat{e}_{t+s} .
\end{aligned}
$$

Now, make the following assumption.

Assumption 2. Kernel weights $w($.) satisfy for all $x \in R,|w(x)| \leqslant 1$ and $w(x)=$ $w(-x) ; w(0)=1 ; w(x)$ is continuous at zero and for almost all $x \in R ; \int_{R}|w(x)| \mathrm{d} x$ $<\infty$. Also, the lag truncation parameter 1 satisfies $l=\mathrm{o}\left(n^{1 / 2}\right)$. 
Theorem 1. If $Y_{t}$ is generated by Eq. (1) and under Assumptions 1 and 2

(a)

$$
\hat{Z}_{\rho} \Rightarrow \frac{\int_{0}^{1} Q_{\alpha} \mathrm{d} Q_{\alpha}}{\int_{0}^{1} Q_{\alpha}^{2}}
$$

(b)

$$
\hat{Z}_{t} \Rightarrow \frac{\int_{0}^{1} Q_{\alpha} \mathrm{d} Q_{\alpha}}{\left(\int_{0}^{1} Q_{\alpha}^{2}\right)^{1 / 2}\left[Q_{\alpha}(1)^{2}-2 \int_{0}^{1} Q_{\alpha} \mathrm{d} Q_{\alpha}\right]^{1 / 2}} .
$$

Theorem 1 is the extension of Theorem 4.1a-b of Phillips and Ouliaris (1990) to the case where the distribution of the disturbances $\varepsilon_{t}$ are in the normal domain of attraction of a symmetric stable law with index $\alpha, 0<\alpha<2$. The results show that the $\hat{Z}_{t}, \hat{Z}_{\rho}$ test statistics for cointegration can be represented as functionals of stable processes in the case where the disturbances have infinite variance, rather than functionals of Brownian motion as in the case of squareintegrable disturbances. Another point to note is the limit law in Theorem $1 \mathrm{~b}$ is a functional of the quadratic variation of $Q_{\alpha}$. Quadratic variation of $Q_{\alpha}$ is equivalent to $\left(Q_{\alpha}(1)^{2}-2 \int_{0}^{1} Q_{\alpha} \mathrm{d} Q_{\alpha}\right)=\kappa^{\prime} \kappa t$. This is also a generalization of the result found by Phillips and Ouliaris (1990). An important point is we could have analyzed the ADF tests of Engle and Granger (1987), but this would have probably resulted in the same limit law as the $\hat{Z}_{t}$ test at the cost of using a more complicated proof.

In Theorem 1 observe that the limit laws only depend on the number of variables $(p)$ in the system and the index of stability $\alpha$. If the statistics are based on a regression with a fitted intercept in Eq. (11) then the limiting distributions are functionals of demeaned stable processes. In the same way, if there is a time trend, in the regression, the limit law in Theorem 1 consists of functionals of detrended stable processes.

Theorem 1 allows us to investigate whether the $\hat{Z}_{\rho}$ and $\hat{Z}_{t}$ tests are robust to infinite variance errors. In this respect, we calculate the size distortions if the conventional critical values in Phillips and Ouliaris (1990) tables are mistakenly used instead of the critical values found in this article. Then we will be able to determine whether Phillips' $\hat{Z}_{\rho}$ and $\hat{Z}_{t}$ tests for cointegration are robust to infinite variance errors or not.

Tables 1 and 2 present estimates of the critical values for the $\hat{Z}_{\rho}$ and $\hat{Z}_{t}$ statistics. ${ }^{1}$ The tables allow for $p(=1,2$ or 3$)$, variables in the system (1). Critical values are provided for the model in Eq. (12) with a constant (left part of

\footnotetext{
${ }^{1}$ Tables 1 and 2 were generated by simulation using 1000 observations with 20,000 iterations. The symmetric stable random variables were generated by the algorithm of Kanter and Steiger (1974).
} 
Table 1

Critical values for $\hat{Z}_{\rho}$ statistic

\begin{tabular}{|c|c|c|c|c|c|c|c|c|}
\hline \multirow[b]{3}{*}{ Size } & \multicolumn{4}{|c|}{ Demeaned } & \multicolumn{4}{|c|}{ Demeaned and detrended } \\
\hline & $\alpha=0.5$ & & & & $\alpha=0.5$ & & & \\
\hline & 0.1 & 0.05 & 0.025 & 0.01 & 0.1 & 0.05 & 0.025 & 0.01 \\
\hline $\begin{array}{l}p=1 \\
p=2 \\
p=3\end{array}$ & $\begin{array}{l}-10.45 \\
-20.61 \\
-30.41 \\
\alpha=1\end{array}$ & $\begin{array}{l}-16.52 \\
-31.09 \\
-46.51\end{array}$ & $\begin{array}{l}-27.62 \\
-47.22 \\
-70.11\end{array}$ & $\begin{array}{r}-53.45 \\
-89.36 \\
-121.11\end{array}$ & $\begin{array}{l}-14.54 \\
-24.40 \\
-33.97 \\
\alpha=1\end{array}$ & $\begin{array}{l}-20.85 \\
-36.40 \\
-51.06\end{array}$ & $\begin{array}{l}-32.51 \\
-55.52 \\
-75.83\end{array}$ & $\begin{array}{r}-62.91 \\
-94.72 \\
-139.46\end{array}$ \\
\hline $\begin{array}{l}p=1 \\
p=2 \\
p=3\end{array}$ & $\begin{array}{c}-10.73 \\
-17.76 \\
-24.33 \\
\alpha=1.5\end{array}$ & $\begin{array}{l}-14.81 \\
-23.37 \\
-31.43\end{array}$ & $\begin{array}{l}-20.54 \\
-30.49 \\
-41.50\end{array}$ & $\begin{array}{l}-30.35 \\
-44.85 \\
-60.40\end{array}$ & $\begin{array}{c}-16.34 \\
-22.75 \\
-29.66 \\
\alpha=1.5\end{array}$ & $\begin{array}{l}-20.92 \\
-29.16 \\
-37.48\end{array}$ & $\begin{array}{l}-27.38 \\
-37.89 \\
-48.62\end{array}$ & $\begin{array}{l}-42.02 \\
-55.30 \\
-72.02\end{array}$ \\
\hline $\begin{array}{l}p=1 \\
p=2 \\
p=3\end{array}$ & $\begin{array}{l}-11.06 \\
-17.14 \\
-23.01\end{array}$ & $\begin{array}{l}-14.26 \\
-21.01 \\
-27.49\end{array}$ & $\begin{array}{l}-17.48 \\
-25.41 \\
-32.49\end{array}$ & $\begin{array}{l}-23.35 \\
-31.97 \\
-39.74\end{array}$ & $\begin{array}{l}-17.48 \\
-22.98 \\
-28.09\end{array}$ & $\begin{array}{l}-21.35 \\
-27.78 \\
-32.91\end{array}$ & $\begin{array}{l}-25.51 \\
-32.89 \\
-38.12\end{array}$ & $\begin{array}{l}-32.19 \\
-40.40 \\
-45.98\end{array}$ \\
\hline
\end{tabular}

Tables 1 and 2) and with a constant term and a trend (right part of Tables 1 and 2). These tests reject the null hypothesis of no cointegration if the test statistic is smaller than the appropriate critical value. For example, when $\alpha=1$, for a regression with a constant term and $p=3$, we reject at the $5 \%$ level if the computed value of $\hat{Z}_{\rho}$ is less than -31.44 . From Tables 1 and 2 it is easy to see that the critical values are larger in absolute value when $\alpha<2$ than their counterparts in Phillips and Ouliaris (1990). This suggests that if the researchers falsely assume that error terms are square integrable the $\hat{Z}_{\rho}, \hat{Z}_{t}$ tests will slightly over-reject the null of no cointegration.

However, comparing critical values by themselves do not indicate the extent of size distortion. So we also calculated the size distortions if the conventional Phillips and Ouliaris (1990) criticals are mistakenly used. The results are given in Tables 5 and $6 .^{2}$ For nearly every case, the actual rejection frequency of the null of no cointegration exceeds the nominal level.

The size distortion generally increases with the number of variables $p$ and as $\alpha$ decreases. For example, if we analyze the demeaned and detrended $\hat{Z}_{\rho}$ statistic with 3 variables in Table 5, the actual rejection frequencies range between 0.066 and $0.022,0.083$ and $0.035,0.103$ and $0.06,0.134$ and 0.11 at the levels of 0.01 ,

\footnotetext{
${ }^{2}$ Tables 5 and 6 were generated using 5000 iterations.
} 
Table 2

Critical values for $\hat{Z}_{t}$ statistic

\begin{tabular}{|c|c|c|c|c|c|c|c|c|}
\hline \multirow[b]{3}{*}{ Size } & \multicolumn{4}{|c|}{ Demeaned } & \multicolumn{4}{|c|}{ Demeaned and detrended } \\
\hline & $\alpha=0.5$ & & & & $\alpha=0.5$ & & & \\
\hline & 0.1 & 0.05 & 0.025 & 0.01 & 0.1 & 0.05 & 0.025 & 0.01 \\
\hline $\begin{array}{l}p=1 \\
p=2 \\
p=3\end{array}$ & $\begin{array}{l}-2.77 \\
-3.50 \\
-4.15 \\
\alpha=1\end{array}$ & $\begin{array}{l}-3.67 \\
-4.45 \\
-5.19\end{array}$ & $\begin{array}{l}-4.83 \\
-5.58 \\
-6.43\end{array}$ & $\begin{array}{l}-7.12 \\
-7.91 \\
-9.04\end{array}$ & $\begin{array}{l}-2.93 \\
-3.68 \\
-4.30 \\
\alpha=1\end{array}$ & $\begin{array}{l}-3.73 \\
-4.66 \\
-5.35\end{array}$ & $\begin{array}{l}-4.92 \\
-5.88 \\
-6.69\end{array}$ & $\begin{array}{l}-7.17 \\
-8.17 \\
-9.33\end{array}$ \\
\hline $\begin{array}{l}p=1 \\
p=2 \\
p=3\end{array}$ & $\begin{array}{r}-2.64 \\
-3.16 \\
-3.64 \\
\alpha=1.5\end{array}$ & $\begin{array}{l}-3.22 \\
-3.74 \\
-4.22\end{array}$ & $\begin{array}{l}-3.95 \\
-4.38 \\
-4.93\end{array}$ & $\begin{array}{l}-5.08 \\
-5.57 \\
-6.35\end{array}$ & $\begin{array}{l}-3.03 \\
-3.51 \\
-3.96 \\
\alpha=1.5\end{array}$ & $\begin{array}{l}-3.56 \\
-4.06 \\
-4.54\end{array}$ & $\begin{array}{l}-4.23 \\
-4.77 \\
-5.28\end{array}$ & $\begin{array}{l}-5.72 \\
-6.19 \\
-6.80\end{array}$ \\
\hline $\begin{array}{l}p=1 \\
p=2 \\
p=3\end{array}$ & $\begin{array}{l}-2.59 \\
-3.07 \\
-3.49\end{array}$ & $\begin{array}{l}-2.98 \\
-3.45 \\
-3.85\end{array}$ & $\begin{array}{l}-3.40 \\
-3.84 \\
-4.23\end{array}$ & $\begin{array}{l}-4.08 \\
-4.44 \\
-4.82\end{array}$ & $\begin{array}{l}-3.08 \\
-3.49 \\
-3.82\end{array}$ & $\begin{array}{l}-3.45 \\
-3.86 \\
-4.16\end{array}$ & $\begin{array}{l}-3.84 \\
-4.25 \\
-4.52\end{array}$ & $\begin{array}{l}-4.51 \\
-5.01 \\
-5.14\end{array}$ \\
\hline
\end{tabular}

$0.025,0.05$ and 0.1 , respectively, when $\alpha$ increases from 0.5 to 1.5 . Continuing with the same test statistic in Table 5 , when $\alpha=1$, if the number of variables increases from 2 to 3 , the calculated size range between 0.031 and $0.041,0.049$ and $0.059,0.072$ and 0.091 and 0.11 and 0.14 at the nominal levels of $0.01,0.025$, 0.05 and 0.1 , respectively. The results of Table 6 are similar to the ones presented in Table 5. We conclude that the size distortions are moderate, and the Phillips $\hat{Z}_{\rho}$ and $\hat{Z}_{t}$ tests for cointegration are mildly robust to infinite variance errors.

As a special case of our results, we can also analyze whether Phillips and Perron (1988) unit root tests are robust to infinite variance errors $(p=1$ in Tables 5 and 6). Even though the limit theory for the infinite variance case was developed by Chan and Tran (1989) and Phillips (1990) the issue of robustness has not been explored. From Tables 5 and 6 we can see that at the $5 \%$ nominal level the actual size ranges between $6 \%$ and $9 \%$ and $5 \%$ and $6 \%$ for the demeaned $\hat{Z}_{t}$ and $\hat{Z}_{\rho}$ tests, respectively. Similar results can be obtained for the other levels. It appears that these tests are also moderately robust to the infinite second moment in the noise.

\section{Likelihood-based tests}

Consider the following VAR model:

$$
\Delta Y_{t}=\sum_{i=1}^{k-1} \Gamma_{i} \Delta Y_{t-i}+\Pi Y_{t-k}+\varepsilon_{t}
$$


Table 3

Critical values for maximum eigenvalue statistic

\begin{tabular}{|c|c|c|c|c|c|c|c|c|}
\hline \multirow[b]{3}{*}{ Size } & \multicolumn{4}{|c|}{ Without linear trend } & \multicolumn{4}{|c|}{ With linear trend } \\
\hline & \multicolumn{4}{|l|}{$\alpha=0.5$} & \multicolumn{4}{|c|}{$\alpha=0.5$} \\
\hline & 0.1 & 0.05 & 0.025 & 0.01 & 0.1 & 0.05 & 0.025 & 0.01 \\
\hline$p-r=1$ & 7.76 & 13.52 & 23.10 & 46.14 & N.A & N.A & N.A & N.A \\
\hline$p-r=2$ & 22.29 & 36.46 & 59.06 & 110.41 & N.A & N.A & N.A & N.A \\
\hline \multirow[t]{2}{*}{$p-r=3$} & 40.75 & 65.17 & 98.64 & 192.40 & N.A & N.A & N.A & N.A \\
\hline & \multicolumn{4}{|l|}{$\alpha=1$} & \multicolumn{4}{|l|}{$\alpha=1$} \\
\hline$p-r=1$ & 6.93 & 10.36 & 15.36 & 27.69 & A.D & A.D & A.D & A.D \\
\hline$p-r=2$ & 15.93 & 22.34 & 32.95 & 53.34 & A.D & A.D & A.D & A.D \\
\hline \multirow[t]{2}{*}{$p-r=3$} & 26.24 & 37.00 & 52.74 & 87.70 & A.D & A.D & A.D & A.D \\
\hline & \multicolumn{4}{|l|}{$\alpha=1.1$} & \multicolumn{4}{|c|}{$\alpha=1.1$} \\
\hline$p-r=1$ & 6.77 & 9.50 & 13.36 & 21.59 & 2.61 & 3.43 & 4.20 & 5.28 \\
\hline$p-r=2$ & 15.03 & 20.27 & 27.72 & 42.31 & 11.95 & 15.46 & 20.52 & 30.38 \\
\hline \multirow[t]{2}{*}{$p-r=3$} & 24.37 & 32.05 & 42.96 & 69.38 & 20.90 & 26.75 & 35.17 & 56.19 \\
\hline & \multicolumn{4}{|l|}{$\alpha=1.5$} & \multicolumn{4}{|c|}{$\alpha=1.5$} \\
\hline$p-r=1$ & 6.61 & 8.74 & 11.34 & 16.36 & 2.63 & 3.58 & 4.60 & 6.05 \\
\hline$p-r=2$ & 13.69 & 16.82 & 20.60 & 28.72 & 12.14 & 14.75 & 17.56 & 23.81 \\
\hline$p-r=3$ & 20.54 & 24.50 & 29.77 & 43.47 & 19.34 & 22.77 & 27.37 & 37.19 \\
\hline
\end{tabular}

Note: AD is available on demand from the author. Since the case for $\alpha=1$ with the time trend results in a different limit, it is not included in this table.

where $\varepsilon_{t}$ 's are $p$-dimensional vectors and satisfy Assumption $1 . Y_{1-k}, \ldots, Y_{0}$ are fixed and the quasi-likelihood function for Eq. (14) will be calculated for given values of these. The parameters $\Gamma_{1}, \ldots, \Gamma_{k-1}$ are unrestricted. The model in Eq. (14) is denoted by $\mathrm{H}_{1}$. Then as in Johansen (1988) the hypothesis of (at most) $r$ cointegrating vectors is

$$
\mathrm{H}_{2}: \Pi=\delta \beta^{\prime},
$$

where $\beta$ and $\delta$ are full rank $p \times r$ matrices which represent the cointegrating vectors and the adjustment coefficients, respectively. In this section, first we conduct inference on the number of cointegrating vectors and in order to test different hypotheses we benefit from quasi-likelihood ratio statistics. Then a limit theorem is derived for these quasi-likelihood-ratio-based tests when the errors in Eq. (14) have infinite variance. So, the limit theory for the trace and maximum eigenvalue test statistics of Johansen $(1988,1991)$ are obtained. Critical values of these test statistics are established via simulation studies. Most 
Table 4

Critical values for trace statistic

\begin{tabular}{|c|c|c|c|c|c|c|c|c|}
\hline \multirow[b]{3}{*}{ Size } & \multicolumn{4}{|c|}{ Without linear trend } & \multicolumn{4}{|c|}{ With linear trend } \\
\hline & \multicolumn{4}{|l|}{$\alpha=0.5$} & \multicolumn{4}{|c|}{$\alpha=0.5$} \\
\hline & 0.1 & 0.05 & 0.025 & 0.01 & 0.1 & 0.05 & 0.025 & 0.01 \\
\hline$p-r=1$ & 7.79 & 13.52 & 23.10 & 46.14 & N.A & N.A & N.A & N.A \\
\hline$p-r=2$ & 25.08 & 39.29 & 62.42 & 116.23 & N.A & N.A & N.A & N.A \\
\hline \multirow[t]{2}{*}{$p-r=3$} & 51.22 & 76.99 & 112.31 & 203.82 & N.A & N.A & N.A & N.A \\
\hline & \multicolumn{4}{|l|}{$\alpha=1$} & \multicolumn{4}{|l|}{$\alpha=1$} \\
\hline$p-r=1$ & 6.93 & 10.36 & 15.36 & 27.69 & A.D & A.D & A.D & A.D \\
\hline$p-r=2$ & 18.68 & 25.38 & 36.18 & 56.76 & A.D & A.D & A.D & A.D \\
\hline \multirow[t]{2}{*}{$p-r=3$} & 36.08 & 47.52 & 63.50 & 98.83 & A.D & A.D & A.D & A.D \\
\hline & \multicolumn{4}{|l|}{$\alpha=1.1$} & \multicolumn{4}{|c|}{$\alpha=1.1$} \\
\hline$p-r=1$ & 6.77 & 9.50 & 13.36 & 21.59 & 2.61 & 3.43 & 4.20 & 5.28 \\
\hline$p-r=2$ & 17.80 & 23.23 & 31.35 & 46.23 & 13.05 & 16.54 & 21.44 & 31.58 \\
\hline \multirow[t]{2}{*}{$p-r=3$} & 34.27 & 42.57 & 53.88 & 82.14 & 28.66 & 34.77 & 43.72 & 64.15 \\
\hline & \multicolumn{4}{|l|}{$\alpha=1.5$} & \multicolumn{4}{|c|}{$\alpha=1.5$} \\
\hline$p-r=1$ & 6.61 & 8.74 & 11.34 & 16.36 & 2.63 & 3.58 & 4.60 & 6.05 \\
\hline$p-r=2$ & 16.42 & 19.90 & 24.22 & 32.22 & 13.21 & 15.89 & 18.65 & 24.79 \\
\hline$p-r=3$ & 30.41 & 34.99 & 40.71 & 54.11 & 27.42 & 31.25 & 36.03 & 45.95 \\
\hline
\end{tabular}

Note: AD is available on demand from the author. Since the case for $\alpha=1$ with the time trend results in a different limit, it is not included in this table.

important of all, the size distortions of these test statistics are calculated when the conventional critical values of Johansen and Juselius (1990) are used rather than the tables in this article. As we know the underlying assumption of the Johansen and Juselius (1990) tables are square-integrable disturbances (i.e. $\alpha=2$ ). However, in this paper infinite variance errors $(\alpha<2)$ are assumed and the tables reflect this. So we are able to determine whether Johansen's trace and maximum eigenvalue test statistics are robust to infinite variance errors or not. Before presenting the notation and main theorem of this section we state an important theorem that will be useful for the subsequent proofs.

Theorem 2. Let $\delta_{\perp}^{\prime} \psi \beta_{\perp}$ have full rank $(p-r)$. Define

$$
C=\beta_{\perp}\left(\delta_{\perp}^{\prime} \psi \beta_{\perp}\right)^{-1} \delta_{\perp}^{\prime} .
$$

Then $\Delta Y_{t}$ and $\beta^{\prime} Y_{t}$ are, given initial distributions, stationary while $Y_{t}$ is nonstationary. If the initial distributions are expressed in terms of the doubly infinite 
sequence then $\Delta Y_{t}$ has a representation

$$
\Delta Y_{t}=C(L) \varepsilon_{t},
$$

with $C(1)=C$.

In the above theorem $\delta_{\perp}$ and $\beta_{\perp}$ are $p \times(p-r)$ matrices of full rank such that $\beta^{\prime} \beta_{\perp}=0, \delta^{\prime} \delta_{\perp}=0$. Denote the characteristic polynomial in Eq. (14) as

$$
\Pi(z)=(1-z) I-\sum_{i=1}^{k-1} \Gamma_{i}(1-z) z^{i}-\Pi z^{k},
$$

$-\psi=\psi(1)$ is the derivative of $\Pi(z)$ for $z=1$.

Now we can introduce some notation in order to clarify the proofs. As in Johansen (1991) let $Z_{0 t}=\Delta Y_{t}, Z_{1 t}=\left(\Delta Y_{t-1}^{\prime}, \ldots, \Delta Y_{t-(k-1)}^{\prime}\right)^{\prime}$ and $Z_{k t}=Y_{t-k}$ and let $\Gamma=\left(\Gamma_{1}, \ldots, \Gamma_{k}\right)$. Then Eq. (15) can be rewritten as

$$
Z_{0}=\Gamma Z_{1 t}+\delta \beta^{\prime} Z_{k t}+\varepsilon_{t}
$$

Define the product moment matrices and the residual sum of squares

$$
\begin{aligned}
& M_{i j}=a_{n}^{-2} \sum_{t=1}^{n} Z_{i t} Z_{j t}^{\prime} \quad(i, j=0,1, k), \\
& S_{i j}=M_{i j}-M_{i 1} M_{11}^{-1} M_{1 j} \quad(i, j=0, k) .
\end{aligned}
$$

Then it can be shown that the maximized quasi-likelihood function is found from

$$
L_{\max }^{-2 / n}=\left|S_{00}\right| \prod_{i=1}^{r}\left(1-\hat{\lambda}_{i}\right)
$$

where $\hat{\lambda}_{i}$ 's are the estimated ordered eigenvalues $\hat{\lambda}_{1}>\cdots>\hat{\lambda}_{p}$ of $S_{k 0} S_{00}^{-1} S_{0 k}$ with respect to $S_{k k}$, (Johansen 1988). Using Eq. (19) the quasi-likelihood ratio test statistic can be set up. First when $r=p ; \mathrm{H}_{2}(r)=\mathrm{H}_{1}$ so that for the hypothesis $\mathrm{H}_{2}(r)$ in $\mathrm{H}_{1}, L_{\max }(r) / L_{\max }(p)$ is the quasi-likelihood ratio test statistics. When the hypothesis is $\mathrm{H}_{2}(r)$ in $\mathrm{H}_{2}(r+1), L_{\max }(r) / L_{\max }(r+1)$ is the corresponding test statistic. Before the main theorem of this section we present the test statistics for cointegration.

The likelihood ratio test statistic for hypothesis $\mathrm{H}_{2}: \Pi=\delta \beta^{\prime}$ versus $\mathrm{H}_{1}$ is given by

$$
L R_{u}=-n \sum_{i=r+1}^{p} \ln \left(1-\hat{\lambda}_{i}\right)
$$


whereas the likelihood ratio test statistic of $\mathrm{H}_{2}(r)$ versus $\mathrm{H}_{2}(r+1)$ is given by

$$
L R_{u}^{*}=-n \ln \left(1-\hat{\lambda}_{r+1}\right) .
$$

The main theorem of this section can now be presented.

Theorem 3. Under Assumption 1

$$
\begin{aligned}
L R_{u} \Rightarrow & \operatorname{tr}\left\{\left[Y_{\alpha, p-r}, Y_{\alpha, p-r}\right]_{1}^{-1} \int_{0}^{1} \mathrm{~d} Y_{\alpha, p-r} Y_{\alpha, p-r}^{\prime}\right. \\
& \left.\times\left(\int_{0}^{1} Y_{\alpha, p-r} Y_{\alpha, p-r}^{\prime}\right)^{-1} \int_{0}^{1} Y_{\alpha, p-r} \mathrm{~d} Y_{\alpha, p-r}^{\prime}\right\},
\end{aligned}
$$

where $Y_{\alpha, p-r}$ is a $(p-r)$-dimensional symmetric stable process with independent components. Then

$$
L R_{u}^{*} \Rightarrow \lambda_{\max }
$$

where $\lambda_{\max }$ is the maximum eigenvalue of the matrix in Eq. (20).

In Eq. (20), $\left[Y_{\alpha, p-r}, Y_{\alpha, p-r}\right]_{1}$ is the quadratic variation of $Y_{\alpha, p-r}$ defined as

$$
\begin{aligned}
{\left[Y_{\alpha, p-r} Y_{\alpha, p-r}\right]_{1}=} & Y_{\alpha, p-r}(1) Y_{\alpha, p-r}(1)^{\prime} \\
& \left.-\left(\int_{0}^{1} Y_{\alpha, p-r} \mathrm{~d} Y_{\alpha, p-r}^{\prime}\right)\right)^{\prime}-\int_{0}^{1} Y_{\alpha, p-r} \mathrm{~d} Y_{\alpha, p-r}^{\prime} .
\end{aligned}
$$

Note that the limit theory for the test statistic in Eq. (20) depends only on the number of nonstationary variables $(p-r)$ in the system and $\alpha$. This theorem generalizes Theorem 3 of Johansen (1988) to the case where $\varepsilon_{t}$ 's have infinite variance. Here the limit theory consists of functionals of symmetric stable processes with independent components rather than functionals of standard Brownian motions as in Johansen (1988). It can also be seen that when the disturbances have infinite variance, the quadratic variation of stable process is included in the limit law. However, when $\alpha=2$, the quadratic variation of the standard Brownian motion is the identity matrix. Both test statistics also can be easily derived when there is a constant term in Eq. (14) but with no linear time trend. Then the functionals will consist of the demeaned symmetric stable processes in the limit law in Theorem 3. The left portion of Tables 3 and 4 present the estimates of the critical values of the maximal eigenvalue and trace statistics of Johansen (1991) under the assumption of infinite variance errors. The model that is used in left part of Tables 3 and 4 is Eq. (14) with an intercept. But the model has no linear trend in the levels of $Y_{t}$. Otherwise the intercept is 
Table 5

Size distortions for $\hat{Z}_{\rho}$ statistic

\begin{tabular}{|c|c|c|c|c|c|c|c|c|}
\hline \multirow[b]{3}{*}{ Size } & \multicolumn{4}{|c|}{ Demeaned } & \multicolumn{4}{|c|}{ Demeaned and detrended } \\
\hline & \multicolumn{4}{|l|}{$\alpha=0.5$} & \multicolumn{4}{|l|}{$\alpha=0.5$} \\
\hline & 0.1 & 0.05 & 0.025 & 0.01 & 0.1 & 0.05 & 0.025 & 0.01 \\
\hline$p=1$ & 0.0895 & 0.0647 & 0.0495 & 0.0372 & 0.0629 & 0.4685 & 0.0367 & 0.0295 \\
\hline$p=2$ & 0.1322 & 0.0972 & 0.0762 & 0.0560 & 0.1064 & 0.0820 & 0.0668 & 0.0528 \\
\hline \multirow[t]{2}{*}{$p=3$} & 0.1676 & 0.1290 & 0.1046 & 0.0828 & 0.1338 & 0.1028 & 0.0828 & 0.0656 \\
\hline & \multicolumn{4}{|l|}{$\alpha=1$} & \multicolumn{4}{|l|}{$\alpha=1$} \\
\hline$p=1$ & 0.0912 & 0.0572 & 0.0387 & 0.0255 & 0.0729 & 0.0463 & 0.0314 & 0.0224 \\
\hline$p=2$ & 0.1100 & 0.0716 & 0.0486 & 0.0314 & 0.0966 & 0.0624 & 0.0444 & 0.0308 \\
\hline \multirow[t]{2}{*}{$p=3$} & 0.1396 & 0.0908 & 0.0588 & 0.0410 & 0.1108 & 0.0730 & 0.0500 & 0.0364 \\
\hline & \multicolumn{4}{|l|}{$\alpha=1.5$} & \multicolumn{4}{|l|}{$\alpha=1.5$} \\
\hline$p=1$ & 0.0958 & 0.0531 & 0.0294 & 0.0151 & 0.0847 & 0.0478 & 0.0271 & 0.0151 \\
\hline$p=2$ & 0.0966 & 0.0570 & 0.0342 & 0.0166 & 0.0964 & 0.0496 & 0.0304 & 0.0164 \\
\hline$p=3$ & 0.1072 & 0.0568 & 0.0310 & 0.0154 & 0.1102 & 0.0606 & 0.0352 & 0.0216 \\
\hline
\end{tabular}

Table 6

Size distortions for $\hat{Z}_{t}$ statistic

\begin{tabular}{|c|c|c|c|c|c|c|c|c|}
\hline \multirow[b]{3}{*}{ Size } & \multicolumn{4}{|c|}{ Demeaned } & \multicolumn{4}{|c|}{ Demeaned and detrended } \\
\hline & \multicolumn{4}{|l|}{$\alpha=0.5$} & \multicolumn{4}{|l|}{$\alpha=0.5$} \\
\hline & 0.1 & 0.05 & 0.025 & 0.01 & 0.1 & 0.05 & 0.025 & 0.01 \\
\hline$p=1$ & 0.1210 & 0.0941 & 0.0757 & 0.0599 & 0.0809 & 0.0649 & 0.0527 & 0.0427 \\
\hline$p=2$ & 0.1412 & 0.1072 & 0.0832 & 0.0662 & 0.1148 & 0.0924 & 0.0734 & 0.0592 \\
\hline \multirow[t]{2}{*}{$p=3$} & 0.1686 & 0.1320 & 0.1098 & 0.0924 & 0.1382 & 0.1034 & 0.0816 & 0.0678 \\
\hline & \multicolumn{4}{|l|}{$\alpha=1$} & \multicolumn{4}{|l|}{$\alpha=1$} \\
\hline$p=1$ & 0.1115 & 0.0767 & 0.0567 & 0.0406 & 0.0867 & 0.0603 & 0.0444 & 0.0329 \\
\hline$p=2$ & 0.1138 & 0.0772 & 0.0550 & 0.0386 & 0.1014 & 0.0692 & 0.0496 & 0.0360 \\
\hline \multirow[t]{2}{*}{$p=3$} & 0.1342 & 0.0848 & 0.0612 & 0.0450 & 0.1111 & 0.0760 & 0.0524 & 0.0388 \\
\hline & \multicolumn{4}{|l|}{$\alpha=1.5$} & \multicolumn{4}{|l|}{$\alpha=1.5$} \\
\hline$p=1$ & 0.1043 & 0.0638 & 0.0396 & 0.0245 & 0.0923 & 0.0546 & 0.0332 & 0.0206 \\
\hline$p=2$ & 0.0966 & 0.0558 & 0.0362 & 0.0204 & 0.0960 & 0.0536 & 0.0306 & 0.0192 \\
\hline$p=3$ & 0.1024 & 0.0544 & 0.0320 & 0.0186 & 0.1082 & 0.0574 & 0.0372 & 0.0236 \\
\hline
\end{tabular}


not restricted. The model with linear time trend will be investigated in the next section. Observe that at the left-most column we have, $p-r$, which are the number of nonstationary variables (i.e. they are not cointegrated with the other variables). This means we have $p$ variables in $Y_{t}$ and at most $r$ of them are cointegrated. The critical values are simulated in the same way as in Tables 1 and 2 . But the different sizes represent the right-tail probabilities of the distribution. These tests reject the null hypothesis of at most $r$ cointegrating vectors in favor of more than $r$ vectors if the computed statistic is larger than the critical value. We illustrate this by an example. In Table 4 assume we have 4 variables in $Y_{t}$ and the null hypothesis is $r=1$ against the alternative of more than 1 cointegrating vector. At $5 \%$ level if our $\alpha=1.5$ then we can reject this hypothesis if the computed statistic is larger than 34.99.

In Table 3, we reject the null hypothesis of $r$ cointegrating vectors for the alternative of $r+1$ cointegrating relations if the statistic is larger than the critical value. We can give an example. If we analyze Table 3 , with no linear trend, using the example in the previous paragraph we can reject the null of 1 cointegrating vector in favor of 2 if the computed statistic is larger than 24.50 .

We can now investigate whether the trace and maximum eigenvalue test statistics of Johansen (1991), without the linear trend, are robust to infinite variance errors or not. For this reason the size distortions of both test statistics that arise from mistakenly using the conventional Johansen and Juselius (1990) critical values are calculated and are given at the left side of Tables 7 and 8. For almost all cases the calculated sizes are above the nominal levels. The size distortion increases with the number of nonstationary variables $(p-r)$ and decreases as $\alpha$ increases. First take the case of 4 variables and the null of one cointegrating vector (i.e. $p=4, r=1$ ). Concentrating on the maximum eigenvalue statistic without the linear trend in Table 7 , we see that the actual levels range between 0.19 and $0.04,0.22$ and $0.06,0.25$ and $0.09,0.30$ and 0.14 for the nominal levels of $0.01,0.025,0.05,0.10$, respectively, when $\alpha$ increases from 0.5 to 1.5. When $\alpha=1$, increasing the number of nonstationary variables $(p-r)$ from 1 to 3 for the maximum eigenvalue statistic without the linear trend at the 0.10 , $0.05,0.025,0.01$ nominal levels we see that the actual levels increase from 0.11 to $0.20,0.08$ to $0.16,0.06$ to $0.13,0.04$ to 0.10 , respectively. Thus, when $\alpha$ is near 1 , Tables 7 and 8 suggest large size distortions. So we can say that Johansen's trace and maximum eigenvalue tests for cointegration are not even mildly robust to infinite variance errors like the residual-based tests studied in Section 3 of this paper.

Note that when $p=1$ and $r=0$, the maximum eigenvalue and trace tests are the square of Dickey-Fuller's $t$-test. From Table 7 we observe that when $\alpha$ is between 0.5 and 1.5 the actual size ranges between $10 \%$ and $6 \%$ for the demeaned maximum eigenvalue statistic at the $5 \%$ level. So there is moderate size distortion for this particular unit root test with infinite variance errors. 
Table 7

Size distortions for maximum eigenvalue statistic

\begin{tabular}{|c|c|c|c|c|c|c|c|c|}
\hline \multirow[b]{3}{*}{ Size } & \multicolumn{4}{|c|}{ Without linear trend } & \multicolumn{4}{|c|}{ With linear trend } \\
\hline & \multicolumn{4}{|l|}{$\alpha=0.5$} & \multicolumn{4}{|l|}{$\alpha=0.5$} \\
\hline & 0.1 & 0.05 & 0.025 & 0.01 & 0.10 & 0.05 & 0.025 & 0.01 \\
\hline$p-r=1$ & 0.1186 & 0.0956 & 0.0779 & 0.0610 & N.A & N.A & N.A & N.A \\
\hline$p-r=2$ & 0.2154 & 0.1812 & 0.1537 & 0.1275 & N.A & N.A & N.A & N.A \\
\hline \multirow{2}{*}{$p-r=3$} & 0.2980 & 0.2563 & 0.2240 & 0.1910 & N.A. & N.A & N.A & N.A \\
\hline & \multicolumn{4}{|l|}{$\alpha=1$} & \multicolumn{4}{|l|}{$\alpha=1$} \\
\hline$p-r=1$ & 0.1063 & 0.0760 & 0.0565 & 0.0414 & A.D & A.D & A.D & A.D \\
\hline$p-r=2$ & 0.1640 & 0.1215 & 0.0934 & 0.0702 & A.D & A.D & A.D & A.D \\
\hline \multirow[t]{2}{*}{$p-r=3$} & 0.2049 & 0.1577 & 0.1278 & 0.1006 & A.D & A.D & A.D & A.D \\
\hline & \multicolumn{4}{|l|}{$\alpha=1.1$} & \multicolumn{4}{|l|}{$\alpha=1.1$} \\
\hline$p-r=1$ & 0.1024 & 0.0695 & 0.0482 & 0.0332 & 0.0842 & 0.0309 & 0.0098 & 0.0026 \\
\hline$p-r=2$ & 0.1500 & 0.1075 & 0.0804 & 0.0586 & 0.0967 & 0.0650 & 0.0473 & 0.0348 \\
\hline \multirow[t]{2}{*}{$p-r=3$} & 0.1876 & 0.1396 & 0.1118 & 0.0837 & 0.1382 & 0.1022 & 0.0755 & 0.0570 \\
\hline & \multicolumn{4}{|l|}{$\alpha=1.5$} & \multicolumn{4}{|l|}{$\alpha=1.5$} \\
\hline$p-r=1$ & 0.0971 & 0.0613 & 0.0388 & 0.0239 & 0.0876 & 0.0391 & 0.0160 & 0.0057 \\
\hline$p-r=2$ & 0.1255 & 0.0825 & 0.0540 & 0.0348 & 0.1013 & 0.0596 & 0.0382 & 0.0233 \\
\hline$p-r=3$ & 0.1375 & 0.0865 & 0.0595 & 0.0386 & 0.1146 & 0.0729 & 0.0481 & 0.0314 \\
\hline
\end{tabular}

After investigating the tables we can conclude that using the critical values of trace and maximum eigenvalue test statistics in Johansen $(1988,1991)$ and Johansen and Juselius (1990) instead of the tables in this paper when $\alpha$ is near 1, will increase the likelihood of rejecting the null of $r$ cointegrating vectors in favor of more cointegrating ones.

\section{Extension to time trend}

In this section we extend the results of the previous section to the quasilikelihood-ratio-based tests with linear time trend. In this respect, the results of this section are the generalization of Theorem 2.1 in Johansen (1991) to the case where the error terms have infinite variance. However, the results derived here cover only the stable processes with the index $\alpha, 1<\alpha<2$. Thus, the limit theory of the likelihood-based tests for cointegration cannot be derived by the technique that is used, when the error term have infinite mean. The case of $\alpha<1$, is not very common even in the case of economic variables of Latin American 
Table 8

Size distortions for trace statistic

\begin{tabular}{|c|c|c|c|c|c|c|c|c|}
\hline \multirow[b]{3}{*}{ Size } & \multicolumn{4}{|c|}{ Without linear trend } & \multicolumn{4}{|c|}{ With linear trend } \\
\hline & \multicolumn{4}{|l|}{$\alpha=0.5$} & \multicolumn{4}{|l|}{$\alpha=0.5$} \\
\hline & 0.1 & 0.05 & 0.025 & 0.01 & 0.10 & 0.05 & 0.025 & 0.01 \\
\hline$p-r=1$ & 0.1186 & 0.0966 & 0.0779 & 0.0661 & N.A & N.A & N.A & N.A \\
\hline$p-r=2$ & 0.2063 & 0.1676 & 0.1456 & 0.1234 & N.A & N.A & N.A & N.A \\
\hline \multirow{2}{*}{$p-r=3$} & 0.2765 & 0.2341 & 0.2024 & 0.1739 & N.A & N.A & N.A & N.A \\
\hline & \multicolumn{4}{|l|}{$\alpha=1$} & \multicolumn{4}{|l|}{$\alpha=1$} \\
\hline$p-r=1$ & 0.1063 & 0.0760 & 0.0565 & 0.0414 & A.D & A.D & A.D & A.D \\
\hline$p-r=2$ & 0.1549 & 0.1165 & 0.0900 & 0.0689 & A.D & A.D & A.D & A.D \\
\hline \multirow[t]{2}{*}{$p-r=3$} & 0.1940 & 0.1484 & 0.1168 & 0.0918 & A.D & A.D & A.D & A.D \\
\hline & \multicolumn{4}{|l|}{$\alpha=1.1$} & \multicolumn{4}{|l|}{$\alpha=1.1$} \\
\hline$p-r=1$ & 0.1024 & 0.0700 & 0.0500 & 0.0300 & 0.0842 & 0.0309 & 0.0098 & 0.0026 \\
\hline$p-r=2$ & 0.1432 & 0.0993 & 0.0764 & 0.0574 & 0.0940 & 0.0630 & 0.0442 & 0.0326 \\
\hline \multirow[t]{2}{*}{$p-r=3$} & 0.1768 & 0.1324 & 0.1038 & 0.0754 & 0.1260 & 0.0900 & 0.0653 & 0.0487 \\
\hline & \multicolumn{4}{|l|}{$\alpha=1.5$} & \multicolumn{4}{|l|}{$\alpha=1.5$} \\
\hline$p-r=1$ & 0.0971 & 0.0613 & 0.0388 & 0.0239 & 0.0876 & 0.0391 & 0.0160 & 0.0057 \\
\hline$p-r=2$ & 0.1220 & 0.0751 & 0.0522 & 0.0353 & 0.0972 & 0.0596 & 0.0345 & 0.0219 \\
\hline$p-r=3$ & 0.1388 & 0.0876 & 0.0569 & 0.0367 & 0.1125 & 0.0684 & 0.0430 & 0.0271 \\
\hline
\end{tabular}

countries. As we rarely encounter infinite mean error terms in economics, this is not a big loss from the practitioners' point. Next, we establish the general model

$$
\Delta Y_{t}=\sum_{i=1}^{k-1} \Delta Y_{t-i}+\Pi Y_{t-k}+\mu+\varepsilon_{t}
$$

This model allows us to have a linear trend in the levels of $Y_{t}$.

We can add two more results to Theorem 2 in this case. First $Y_{t}$ is nonstationary with linear trend $\tau t=C \mu t$. Then if the initial distributions are expressed in terms of the doubly infinite sequence then $\Delta Y_{t}$ has the following representation:

$$
\Delta Y_{t}=C(L)\left(\mu+\varepsilon_{t}\right) .
$$

Given the model (21), the likelihood ratio test statistic for hypothesis $\mathrm{H}_{2}=\delta \beta^{\prime}$ versus $\mathrm{H}_{1}$ is given by

$$
L R_{u}=-n \sum_{i=r+1}^{n} \ln \left(1-\hat{\lambda}_{i}\right),
$$


whereas the likelihood ratio test statistic of $\mathrm{H}_{2}(r)$ versus $\mathrm{H}_{2}(r+1)$ is given by

$$
L R_{u}^{*}=-n \ln \left(1-\hat{\lambda}_{r+1}\right)
$$

Theorem 4. When $1<\alpha<2$ and $\delta_{\perp}^{\prime} \mu \neq 0$ then under Assumption 1

$$
L R_{u} \Rightarrow \operatorname{tr}\left\{\left[Y_{\alpha, p-r}, Y_{\alpha, p-r}\right]_{1}^{-1} V^{\prime} U^{-1} V\right\},
$$

where

$$
V=\int_{0}^{1}\left[\left(\begin{array}{c}
Y_{\alpha, p-r-1}-\int_{0}^{1} Y_{\alpha, p-r-1} \\
t-\frac{1}{2}
\end{array}\right) \mathrm{d} Y_{\alpha, p-r}^{\prime}\right],
$$

and

$$
U=\int_{0}^{1}\left[\begin{array}{c}
Y_{\alpha, p-r-1}-\int_{0}^{1} Y_{\alpha, p-r-1} \\
t-\frac{1}{2}
\end{array}\right]\left[\begin{array}{c}
Y_{\alpha, p-r-1}-\int_{0}^{1} Y_{\alpha, p-r-1} \\
t-\frac{1}{2}
\end{array}\right]^{\prime} .
$$

and $Y_{\alpha, p-r-1}$ is the $p-r-1$ dimensional symmetric stable vector with independent components. Next

$$
L R_{u}^{*} \Rightarrow \lambda_{\max }
$$

where $\lambda_{\max }$ is the maximum eigenvalue of the matrix in Eq. (22).

Remark. When $\alpha<1$, the linear trend is dominated by the stochastic partial sums so we cannot develop the limit laws. However, at $\alpha=1$ it can be easily shown that $V$ and $U$ in Theorem 4 can consist of a $(p-r+1)$ vector which has a $(p-r)$-dimensional symmetric stable vector and a time trend, instead of the $(p-r)$ vector which has a $(p-r-1)$ symmetric stable vector and the time trend in the case of $1<\alpha<2 .^{3}$ The proof, the table values and size distortions for $\alpha=1$ is available from the author on demand.

Note that the asymptotic theory is different from Johansen (1991). We have the inverse of the quadratic variation of a $(p-r)$ dimensional stable process with symmetric independent components in the limit law in Eq. (22). Similar to the previous section the limit law is represented as functionals of symmetric stable processes rather than the functionals of standard Brownian motions in Theorem 2.1 of Johansen (1991). We also calculated critical values for $\alpha=1.1$ and 1.5 by simulation. The right side of Tables 3 and 4 present the critical values of the maximum eigenvalue and trace statistics, with linear trend, under the assumption of infinite variance errors. The usage of the tables are the same as

\footnotetext{
${ }^{3}$ I owe this suggestion to an anonymous referee.
} 
the tables that are presented in Section 4. We calculated size distortions for these test statistics. These are tabulated on the right side of Tables 7 and 8 . However, we do not have the results for $0<\alpha \leqslant 1$, when we have a linear trend. So, instead, we calculated critical values and size distortions when $\alpha=1.1$ and $\alpha=1.5$. Here, if we analyze the right side of Tables 7 and 8 we see that again the size distortions increase with $(p-r)$ and decrease as $\alpha$ increases. But this time the magnitude of the distortions are not as large as the ones cited in Section Section 4 when $\alpha$ changes. For example, if we analyze Table 7 with linear trend we see that when $\alpha=1.1$ with 3 nonstationary variables, the calculated level is $10 \%$ and when $\alpha=1.5$ this is $7 \%$ at the $5 \%$ nominal level. One reason why the size distortion is reduced when the linear trend is included is as follows. When $\alpha$ decreases there are heavier tails for the noise. Then it might become difficult to identify the cointegration relations. If there are deterministic trends, since they are much less varying it could be easier to identify the correct cointegration relation despite the misspecification of the noise process. ${ }^{4}$ However, using the same example but analyzing the case without linear trend we see that the actual level decreases from $14 \%$ to $9 \%$ at the $5 \%$ nominal level when $\alpha$ increases from 1.1 to 1.5 . The same pattern can be seen at the other levels in Tables 7 and 8 .

\section{Conclusions}

This paper generalizes the econometrics literature on the tests for cointegration. Instead of the usual assumption of finite variance errors we analyze the residual-based tests and quasi-likelihood ratio tests for cointegration under the assumption of infinite variance errors. The limit laws consist of functionals of symmetric stable processes rather than Brownian motion. Furthermore, the limit laws depend on ' $\alpha$ ' the index of stability. We should also note that the limit laws that are found for the Johansen's tests are not mere replacements of Brownian motions with symmetric stable laws. They also involve the quadratic variation of a symmetric stable process.

We also check whether the $\hat{Z}_{\rho}$ and $\hat{Z}_{t}$ tests of Phillips and Ouliaris (1990) and the trace and maximum eigenvalue statistics of Johansen (1991) are robust to infinite variance errors. In order to analyze this problem the size distortion induced by mistakenly using the conventional critical values tabulated in Phillips and Ouliaris (1990) and Johansen and Juselius (1990) are calculated. It is observed that the $\hat{Z}_{\rho}$ and $\hat{Z}_{t}$ tests exhibit moderate size distortion when $\alpha$ is small. However, when $\alpha$ is near 1 the trace and maximum eigenvalue tests for cointegration display meaningful size distortion. So in this one dimension we

\footnotetext{
${ }^{4}$ I owe this explanation to an anonymous referee.
} 
find that the residual-based tests of Phillips and Ouliaris (1990) are more robust to infinite variance errors than the quasi-likelihood ratio tests of Johansen (1988, 1991).

We would like to comment on the issues related to the empirical implications of the work. Since the limit laws depend on $\alpha$ we should have an estimate of the stability index in order to conduct these tests. Recently, McCulloch (1996a) finds that estimating $\alpha$ 's with only moderate samples from the tails results in larger estimate of $\alpha$ compared with the true value. He proposes a maximum-likelihood method to estimate $\alpha$ with full sample which gives reliable estimates. Another point that is important to remember is in the data different variables may have different $\alpha$. Unfortunately, there has not been any theoretical result established with different $\alpha$ 's Caner (1997) provides a result for only iid case). Here the conservative strategy may be to choose the minimum of the estimates so that we can avoid size distortions. This strategy is easier to implement in the case of the residual-based tests.

We can say that the results obtained by the trace and maximum eigenvalue tests on the developing country data sets should be evaluated with caution. Since we know by Koedijk and Kool (1992) and Akgiray et al. (1988) that foreign exchange returns in the developing countries are governed by stable laws with stability indexes which range between 1 and 1.5, if the existing studies have found cointegration among variables in the system then this might be easily due to assuming wrongly that $\alpha=2$. Note that even the moderate size distortions associated with $\alpha$ near 2 can change the results of the tests for cointegration. For example if we reject the null of no cointegration in the borderline by assuming finite variance errors, even though the true $\alpha$ is below 2,this result could be easily due to the size distortion. So even in the US where the financial asset returns may be governed by stable laws we should be careful in evaluating the results from the likelihood-ratio-based tests. (McCulloch (1996a,b))

\section{Acknowledgements}

I would like to thank Bruce Hansen for excellent advice, encouragement and comments. I would also like to thank Seminar participants in Brown, Indiana University, ESEM 96 and the associate editor and the two anonymous referees for their comments.

\section{References}

Akgiray, V., Booth, G.G., Seifert, B., 1988. Distribution properties of Latin American black-market exchange rates. Journal of International Money and Finance 7, 37-48.

Caner, M., 1997. Weak convergence to a matrix stochastic integral with stable processes. Econometric Theory 13, 506-529. 
Chan, N.H., Tran, L.T., 1989. On the first order autoregressive process with infinite variance. Econometric Theory 5, 354-362.

Engle, R.F., Granger, C.W.J., 1987. Cointegration and error correction representation, estimation and testing. Econometrica 55, 251-276.

Fama, E.F., 1965. The behavior of stock market prices. Journal of Business 38, 34-105.

Hansen, B.E., 1992. Efficient estimation and testing of cointegrating vectors in the presence of deterministic trends. Journal of Econometrics 53, 87-121.

Johansen, S., 1988. Statistical analysis of cointegration vectors. Journal of Economic Dynamics and Control 12, 231-254.

Johansen, S., 1991. Estimation and hypothesis testing of cointegration vectors in gaussian vector autoregressive models. Econometrica 59, 1551-1580.

Johansen, S., Juselius, K., 1990. Maximum likelihood estimation and inference on cointegrationwith applications to the demand for money. Oxford Bulletin of Economics and Statistics 59, 169-210.

Kanter, M., Steiger, W.L., 1974. Regression and autoregression with infinite variance. Advances in Applied Probability 6, 768-783.

Koedijk, K., Kool, C.J.M., 1992. Tail estimates of East European exchange rates. Journal of Business and Economic Statistics 10, 83-96.

Mandelbrot, B.B., 1963. The variation of certain speculative prices. Journal of Business 36, 394-419.

Mandelbrot, B.B., 1967. The variation of some other speculative prices. Journal of Business 40, 394-413.

McCulloch, J.H., 1996a. Measuring tail thickness in order to estimate the stable index $\alpha$ : a critique. Journal of Business and Economic Statistics, forthcoming.

McCulloch, J.H., 1996b. Financial Applications of Stable Distributions. Handbook of Statistics, vol. 14.

Phillips, P.C.B., 1986. Understanding spurious regressions in econometrics. Journal of Econometrics 6, 768-783.

Phillips, P.C.B., 1987. Time series regression with a unit root. Econometrica 55, 277-301.

Phillips, P.C.B., 1990. Time series regression with a unit root and infinite variance errors. Econometric Theory 6, 44-62.

Phillips, P.C.B., 1995. Robust nonstationary regression. Econometric Theory 11, 912-952.

Phillips, P.C.B., Hansen, B.E., 1990. Statistical inference in instrumental variables regression with I(1) processes. Review of Economic Studies 53, 473-495.

Phillips, P.C.B., Ouliaris, S., 1990. Asymptotic properties of residual-based tests for cointegration. Econometrica 58, 165-193.

Phillips, P.C.B., Perron, P., 1988. Testing for a unit root in time series regression. Biometrika 75, 335-346.

Resnick, S.I., 1986. Point processes, regular variation and weak convergence. Advances in Applied Probability 18, 66-138.

Samorodnitsky, G., Taqqu, M., 1994. Stable Non-Gaussian Random Processes. Chapman \& Hall, New York. 